\title{
Looking for fine structures in galaxies ${ }^{\star}$
}

\author{
M. Faúndez-Abans and M. de Oliveira-Abans \\ CNPq/Laboratório Nacional de Astrofísica, Caixa Postal 21, CEP: 37.500-000, Itajubá, MG, Brazil
}

Received April 11; accepted July 21, 1997

\begin{abstract}
In order to enhance fine structure in direct CCD images of galaxies, transform processing has been employed: working in the transform domain, transform functions have been created and simple high- and low-pass filters have been applied to the images during the process. The results presented herein refer to a test sample of four galaxies: ESO 143-G7, HRG 54103, NGC 5193/5193A, and NGC 5761. It is also discussed the use of a Fourier-hologram image as a transfer function.
\end{abstract}

Key words: galaxies: ESO 143-G7; HRG 54103, NGC 5193/5193 A; NGC 5761 — techniques: image processing; mathematical methods

\section{Introduction}

Image enhancement can be one of the most important tools in the processing of large data sets, making the results more suitable for classification than the original data are as, for example, two-dimensional CCD images of galaxies. One possible approach for image enhancement can be subdivided into two categories: the spatial processing, in which the pixels in the image are manipulated directly, and the transform on the spatial frequency domain, where the image function is first transformed to the transform domain and then processed to meet a specific problem or behavior requirements. After this processing, the inverse transform is needed to yield the final spatial image results.

From an intuitive point of view, an image comprises both high and low spatial frequencies, where the high spatial frequencies correspond to sharp edges and the low spatial frequencies correspond to those regions of approximately uniform gray level.

Pioneering works on studying the observed structure of some galaxies have already been done, even in the

Send offprint requests to: M. Faúndez-Abans

(mfaundez@lna.br)

* Based on observations made at the Observatório do Pico dos Dias, CNPq/Laboratório Nacional de Astrofísica, Brazil; and at Las Campanas Observatory, Chile. mid-century as, for example, Danver's (1942) study of the mathematical form of the spiral arms in galaxies, the Fourier analysis of spiral observed structures by Kalnajs (1974), and Aoki et al. (1979), who predicted that multiarmed modes should grow in some galaxies in addition to two-armed spirals, and suggested that the coexistence of several spiral waves is possible. Spectral analysis of spiral and bar structures in galaxies were developed through two-dimensional Fourier analysis by Iye et al. (1982) and Iye (1983). Fourier transforms on H II distribution and also on spiral component in galaxies have also been performed by Considère \& Athanassoula (1982 and 1988, respectively, and references quoted therein; see also Considère 1980).

Likewise, filtering is one of the most common processes used in the transform domain for image enhancement. The filtering method is applicable to problems such as the recognition of finger prints and visual filtering of letter identification as Solomon \& Pelli's (1994) and Secker's (1995) works, the latter having applied ring median filter on digital images (see also Harris et al. 1983). Sulentic et al. (1985) have pointed out the advantages and disadvantages of spatial frequency filtering in comparison to nonlinear domain tools, e.g., median, modal, and mean filters. The number of textbooks on image processing and restoration is not depreciable; the reader is referred to Marion (1991) and Bates \& McDonnell (1989).

In this work the idea of spatial filtering to find fine structures in galaxies is tested. The computing employs some packages of IRAF and STSDAS and those operations are quickly and easily done. The filters to create the transfer function are simple forms of high- and lowpass filters. The creation of a Fourier hologram image to be used as transfer function after simple filtering is briefly discussed.

\section{Observational approach}

The objects selected for this work (see Table 1) have been extracted from a sample of Ring and Elliptical galaxies of which structural properties like surface brightness and color distributions, morphology, and fine structure are being thoroughly studied. The observations were performed 
at the $1.6 \mathrm{~m}$-telescope of the Observatório do Pico dos Dias of the Laboratório Nacional de Astrofísica, Brazil, with direct CCD camera and BVRI Kron-Cousins filters, and the $1 \mathrm{~m}$-telescope at Las Campanas Observatory, Chile, also direct CCD images with $g$ and $r$ Gunn filters. For each object, only results for one filter will be presented, which suffices for the purposes of this work. Complete results will appear in forthcoming papers.

All the images quoted in Table 1 are the mode of at least 3 frames, and have been pre-processed with IRAF and STSDAS packages, cleaned as much as possible by combination of multiple images, subtraction of cosmic rays, bad pixels, and stars, and correction for background gradients and sky contribution. The removal of the sky background has also the effect of smoothing the sudden discontinuity in flux level at the borders of the image, thus reducing the introduction of artifact when filtering in the linear domain. The possibility of spurious features due to the truncation of the spatial frequency power spectrum remains, nonetheless. The combination of frames suppresses artificial faint haloes around the filtered images due to the reduced background noise.

\section{Image enhancement}

The problem of detecting and enhancing structures in galaxies, such as shells, ripples and $x$-structures is not new. Some simple techniques can be used: blurring the original image by convolution with a kernel (median, modal, gaussian or rectangular) and further subtraction of this new image from the original one to find features otherwise hidden in the overall signal of the galaxy (as dust lanes) or model subtraction, as in the works of Jedrzejewski (1987) or Reduzzi et al. (1994). From our point of view, the search for structures in images is more a matter of applying several techniques to extract as much information as possible, than preferring this or that method.

Some techniques of filtering in the transform domain to obtain image enhancement are described in what follows.

\subsection{Enhancement by transform processing}

In practice, the observed image $g_{i}(x, y)$ can be described by two additive terms:

$g_{i}(x, y)=g_{0}(x, y)+n(x, y)$

where $g_{0}(x, y)$ denotes the hypothetically noise-free intensity image and $n(x, y)$ is the noise. Noise can be estimated if one knows the sources, or by a statistical analysis of a region known to contain a nearly constant gray level; then it can be minimized by classical statistical filtering or by spatial ad hoc processing techniques.

A good intuitive approach to image enhancement is the clustering specification inspired in the suggestion of Toney (1983). It follows from what we generally expect from a processed image: object-distinctive and detailsdiscernible. In the first one, it is expected that all the desired objects are included in the processed image and, in addition, those separate objects are as distinct from one another as possible. In the second one, which is the interesting point of this work, fine details of the objects are expected to be discernible as well as possible. Before starting the analysis, a subimage containing the object of interest copied out from the clean image is created, and which will be the initial input image function $g_{i}(x, y)$.

To apply a spatial filter to the input image we multiply the Fourier transform of the input image, $G_{i}\left(f_{x}, f_{y}\right)$, by another function $H\left(f_{x}, f_{y}\right)$, which defines a linear spatial filter, normally called the transfer function of the filter (see Bow 1984 and references quoted therein). Their product

$G_{\mathrm{t}}\left(f_{x}, f_{y}\right)=G_{i}\left(f_{x}, f_{y}\right) H\left(f_{x}, f_{y}\right)$

is the Fourier transform of the output of the adopted filter. The final image $g_{f}(x, y)$, " the expected processed image", is obtained by the inverse Fourier transform of $G_{\mathrm{t}}$ :

$g_{f}(x, y)=F^{-1}\left[G_{\mathrm{t}}\left(f_{x}, f_{y}\right)\right]$

As well pointed out by Bow (1984), the proper choice of the filter is largely problem-dependent. He has shown that for the high-pass filters Butterworth, exponential, and trapezoidal, the high-frequency-component emphasis increases in the above order, but the preservation of lowfrequency information increases in the reverse order.

The results of two such filtering operations, obtained for a low-pass filter for the transfer function,

$H\left(f_{x}, f_{y}\right)=\left\{\cos \left[\pi G_{i}\left(f_{x}, f_{y}\right)\right]\right\}^{10}$

and the high-pass filter, with the transfer function,

$H\left(f_{x}, f_{y}\right)=2.0-\left\{\cos \left[\pi G_{i}\left(f_{x}, f_{y}\right)\right]\right\}^{20}$

are here presented for illustrative purposes.

We have arbitrarily raised the cosines to the 10th and 20th power in order to make the transfer function fall off quite rapidly from its maximum value of 1 to its minimum value of 0 .

The original image in the filter Gunn $g$ of the peculiar S0 galaxy NGC 5761 is displayed in Fig. 1. The long bridge which is probably a remnant of the interaction of the two upper objects is clearly seen. The almost radial spikes that appear to surround NGC 5761 are real features and extend to the central regions of the galaxy. Several median filters have been applied to the original image in an attempt to enhance as much details as possible with this method. Figure 2 displays the residual image of one of such experiments, made with a kernel of $15 \times 15$ pixels; analyzing all the experiments, it can be seen that the aforementioned spikes do extend to the galactic center and a brighter curved structure connects the bridge to the 
Table 1. The observed objects

\begin{tabular}{lcccclc}
\hline Object & $\alpha_{1950}$ & $\delta_{1950}$ & Telescope & Filter & Type & Ref. \\
\hline HRG 54103 & 011619.2 & -195317.3 & $1.6 \mathrm{~m}$ OPD & $B$ & SA(r)O $)^{+}$ & $1,2,3$ \\
NGC 5193/5193A & 132902.9 & -325838.0 & $1.0 \mathrm{~m} \mathrm{LCO}$ & Gunn $g$ & Ep/S0 & 2 \\
NGC 5761 & 144618.0 & -201012.0 & $1.0 \mathrm{~m} \mathrm{LCO}$ & Gunn $g$ & S0 pec & 2 \\
ESO 143-G7 & 200157.0 & -602112.0 & $1.6 \mathrm{~m} \mathrm{OPD}$ & $B$ & (R)SAB(rs)ab & 1,2 \\
\hline
\end{tabular}

center. Figure 3 displays NGC 5761 after the application of Eq. (5). We have tested the high-pass filter for introduction of texture artifacts by use of a random-noise image, but no such effect has been encountered. It has also been applied to a section of the original image containing the upper left sky portion only, and again no directional artifacts have been created on the sky (see Lorre \& Gillespie 1980). This figure also displays the radial spike-like patterns of Figs. 1 and 2, some knotty structures around the bulge and along the bridge, including the curved "arm" of ESO 580-G38. No other structures stand out.

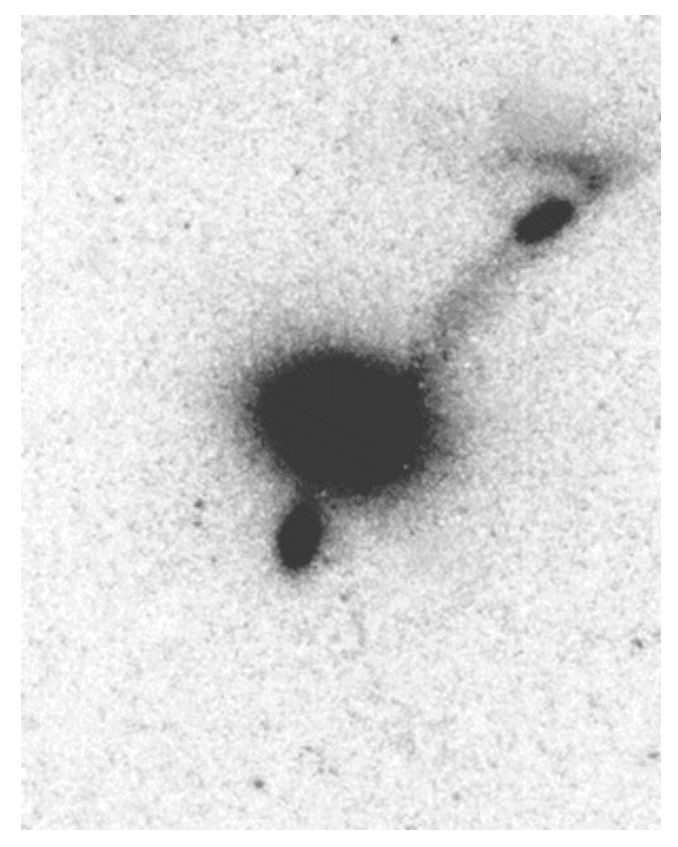

Fig. 1. The Gunn $g$ original image of NGC 5761 (center), ESO 580-G38 (top), and ESO-LV 580-G391 (bottom). In the figure, North is to the bottom and East is on the left

The original image of NGC 5193/5193A is displayed in Fig. 4 , and the residual image of the subtraction of a $5 \times 5$ pixel median is shown in Fig. 5. Most of the radial spikes in the elliptical are artifacts, but the warped disk-like feature in the lenticular galaxy is real, as well as those elliptical's spikes that are parallel to this feature. We have applied the same filtering procedure of above and then enhanced the

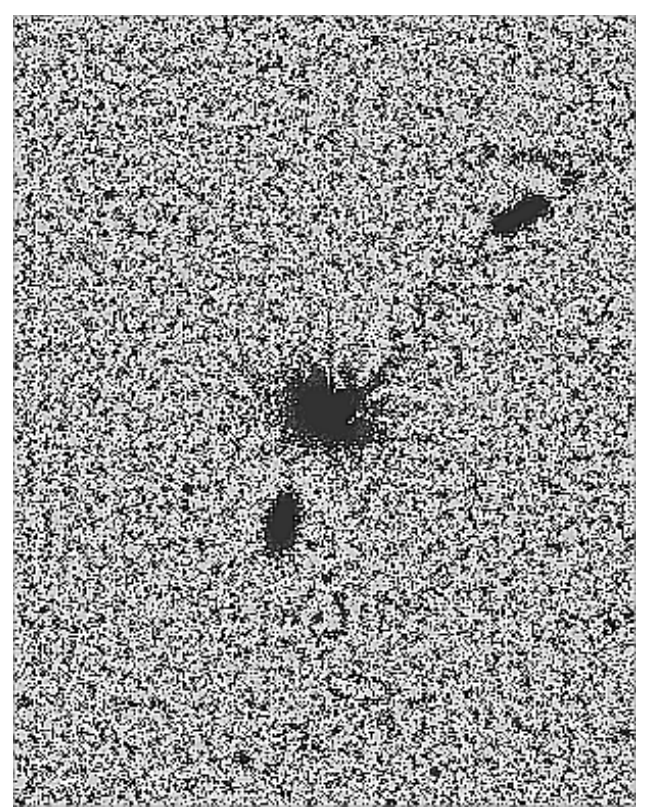

Fig. 2. Median filtering of NGC 5761. The vertical line is a chip flaw enhanced by the process. Note the curved structure on the top and the radial spike-like features in NGC 5761. A probable dusty lane below the region where the bridge encounters the inner part of the bulge is discernible

results by the application of a $10 \times 10$-pixel median filter. Figure 6 displays the results, where the warped dusty disk and the parallel spikes have been well enhanced against a less noisy background.

Another example of this processing is the case of ESO 143-G7, of which original $B$ image is in Fig. 7. The most revealing median filter applied is the one with a $20 \times 20$ pixel kernel, whose residual image is in Fig. 8; the small rim on the far right has been enhanced as well as the extremities of the arms and some knotty features around the bulge. The high-pass filtering (Fig. 9) has enhanced not only the aforementioned rim, but also an extension of if and an interesting bubble-like feature closer to the bulge (a large supernova bubble? two pseudo-oscillations of a thin disk?). The application of a low-pass transfer function suggests the existence of a thin pseudo-bar connecting the starting points of both principal arms of the galaxy, but 


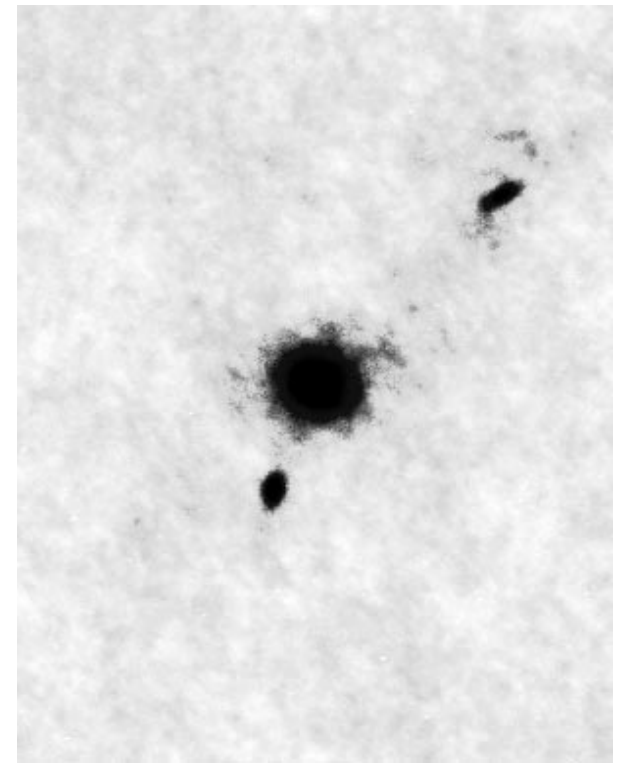

Fig. 3. High-pass filtering of NGC 5761. The chip flaw appear as a blob on top of NGC 5761, but the other features around it are real - see text. A probable curved counterpart of the arm of ESO 580-G38 appears below its boxy bulge. Linear segments appear on both sides of NGC 5761; the one on the right is probably related to the connection with the bridge

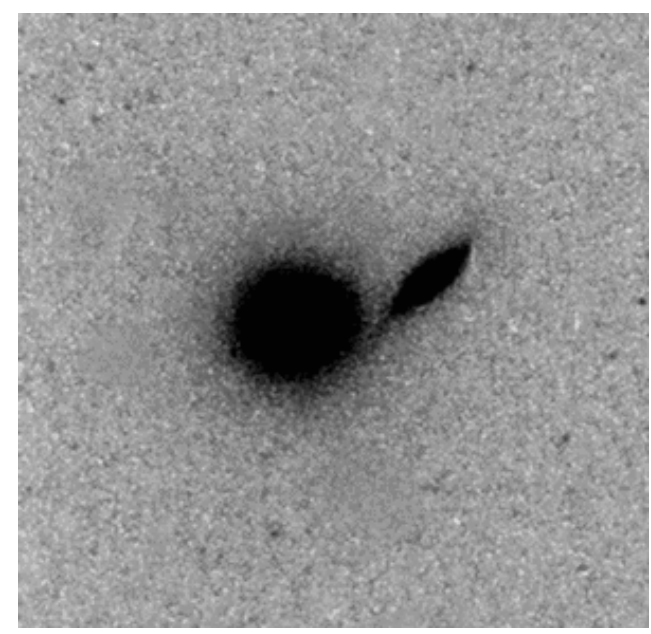

Fig. 4. The Gunn $g$ original image of NGC 5193/5193A. North is at the bottom and East is on the left

the resolution of the image is poor and hence is has not been shown here.

\subsection{Hybrid transfer function}

It is possible to create as many transfer functions as one wishes, applying them to images previously processed or not. Multiple filtering can also be made. We present here the results of first applying Eq. (5) to the original image of HRG 54103 (Fig. 10), and then adopting this new image as the transfer function to be used in Eq. (2). The pro-

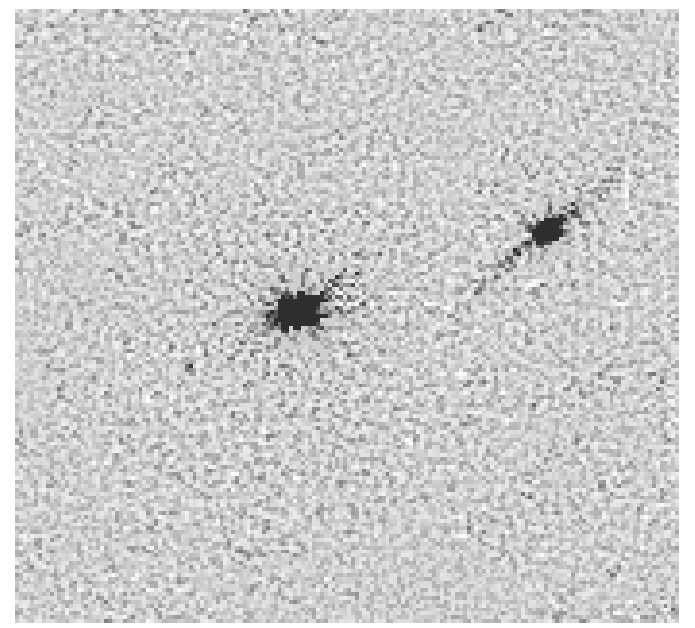

Fig. 5. The residual image of the subtraction of the $5 \times 5$-pixel median-filtered image of NGC 5193/5193A from the original. Note the warped disk seen edge-on and the spikes in NGC 5193 parallel to it

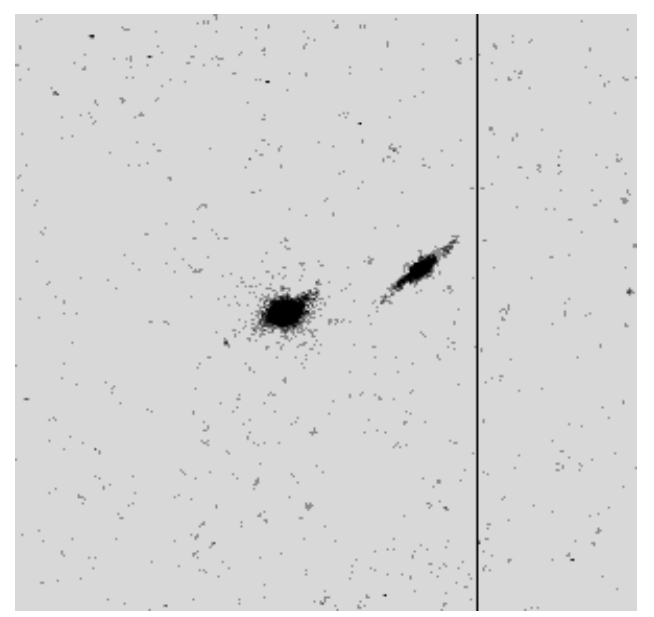

Fig. 6. The high-pass filtering of NGC 5193/5193A, enhanced by the subtraction of its $10 \times 10$-pixel median filtered image. Again, the disk and the parallel spikes have been enhanced, but a little better defined than in Fig. 5. The vertical line is a chip flaw

cessed image is shown in Fig. 11. In this figure one can see two knots, which we associate to satellites in the internal periphery of the bulge of the galaxy. These two objects could be the observable examples of the experiments in numerical simulation of satellite systems such as globular clusters or dwarf galaxies moving within a galaxy, as in the work of Cora et al. (1996). We roughly estimate that each knot is 0.02 of the bulge frame area. On the opposite side of the bulge a small plume-like feature is seen (an internal bar?). A detailed analysis of the physical properties of this object will appear in a future work (FaúndezAbans \& de Oliveira-Abans 1998). Finally, manipulating the LUTs of the image display tool, it is possible to discern 


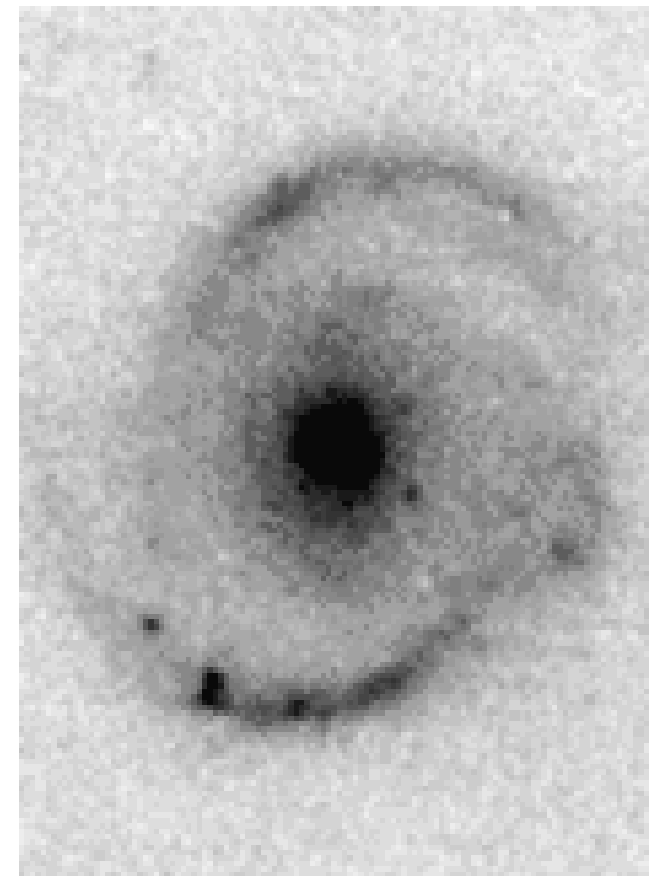

Fig. 7. The $B$ original image of ESO 143-G7

a bar, a thin rim which encapsulates the nucleus, satellite knots and the plume. In Fig. 12 an attempt to enhance this rim by a high-pass filter in the $B$ image is displayed. The contrast has been set to emphasize the pseudo-ring surrounding the nuclear region (better seen on the left side of the image); features such as the bulge, the outer ring, and a faint halo are still discernible. For the sake of comparison, Fig. 13 displays the residual of the subtraction of a $15 \times 15$-pixel median image from the original one. The "rim" that connects the lower part of the external ring to the bulge on the right has been emphasized, as well as the knots on this ring; the white features are artifacts introduced by this particular choice of the median kernel.

\subsection{The Fourier hologram}

Another alternative that has been tested for is to adopt a Fourier hologram as transfer function. The Fourier hologram is described by:

$$
\begin{aligned}
g_{h}\left(f_{x}, f_{y}\right)=a^{2}+\left|G_{i}\left(f_{x}, f_{y}\right)\right|^{2} & +a \mathrm{e}^{i k \theta y} G_{i}\left(f_{x}, f_{y}\right) \\
& +a \mathrm{e}^{-i k \theta y} G_{i}^{*}\left(f_{x}, f_{y}\right)
\end{aligned}
$$

where $g_{h}\left(f_{x}, f_{y}\right)$ is the pixel intensity, $a$ the amplitude, $\left|G_{i}\left(f_{x}, f_{y}\right)\right|^{2}=G_{i}\left(f_{x}, f_{y}\right) G_{i}^{*}\left(f_{x}, f y\right), \quad k=2 \pi / \lambda, \lambda$ is a monochromatic wavelength, $\theta$ is the inclination angle of the reference wave to the holographic plane, and $y$ the adopted maximum amplitude of the holographic image in this plane. This algorithm is a traditional example of a holographic recording set-up for an optical instrument for image formation.

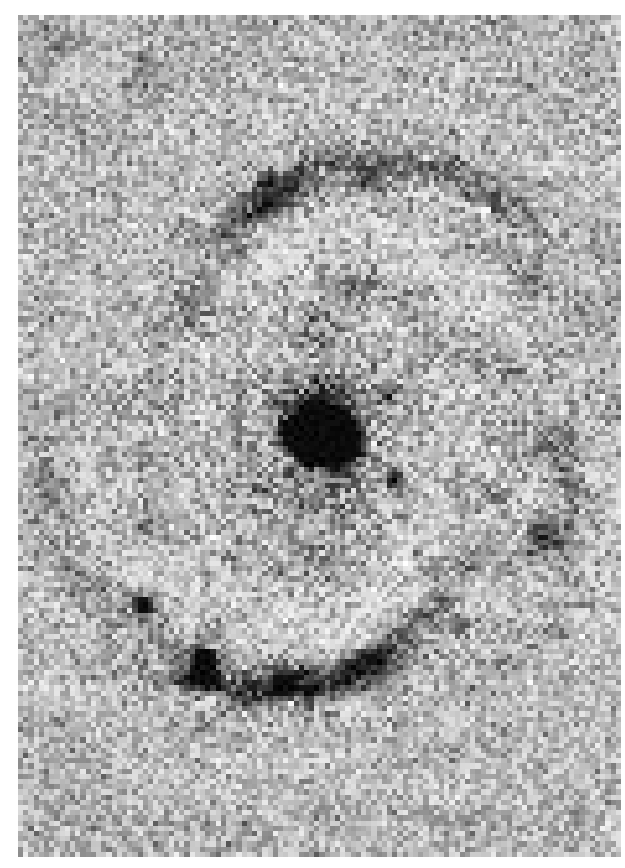

Fig. 8. Result of the subtraction a $20 \times 20$-pixel median image from the original one - see text

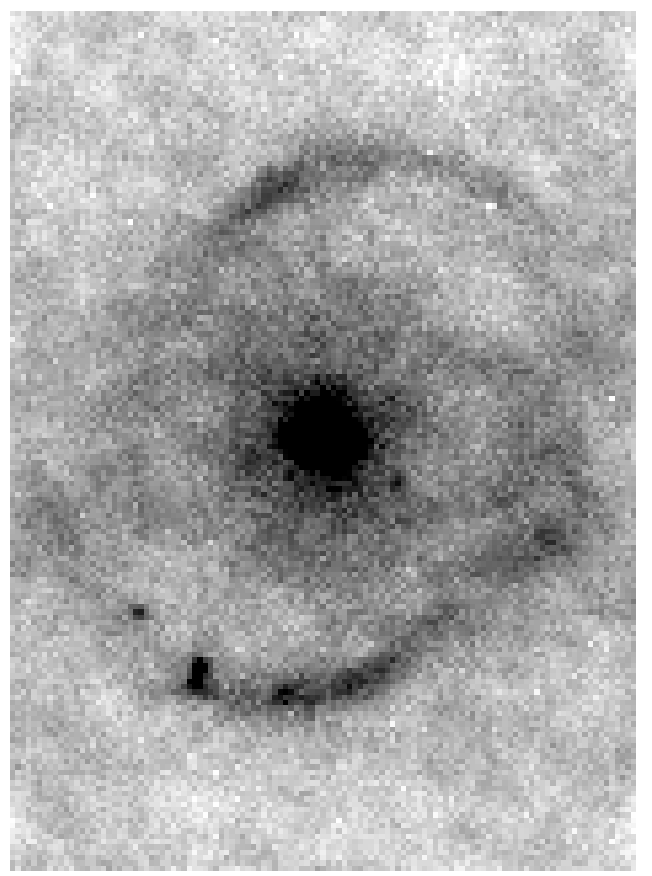

Fig. 9. The high-pass filtering of ESO 143-G7. The spiral structure is preserved, enhancing other structures inside the spiral pattern 


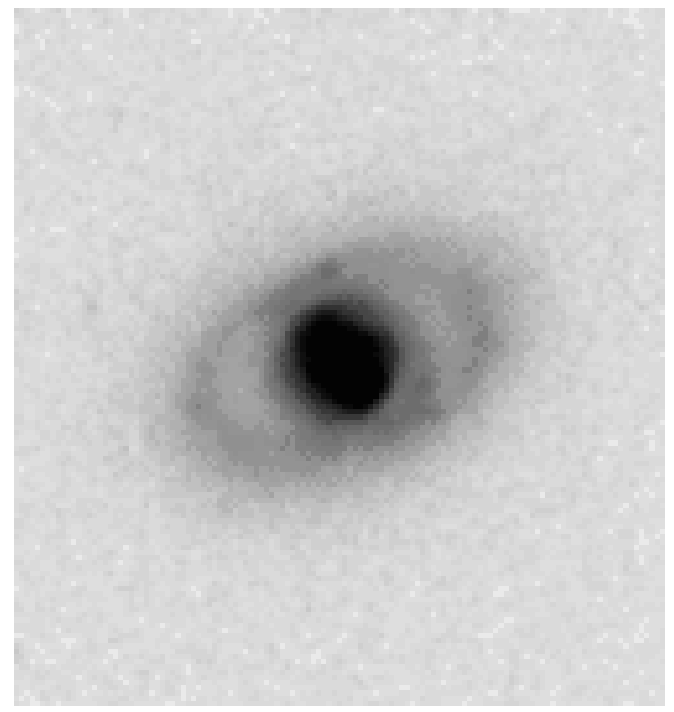

Fig. 10. The original image of HRG 54103

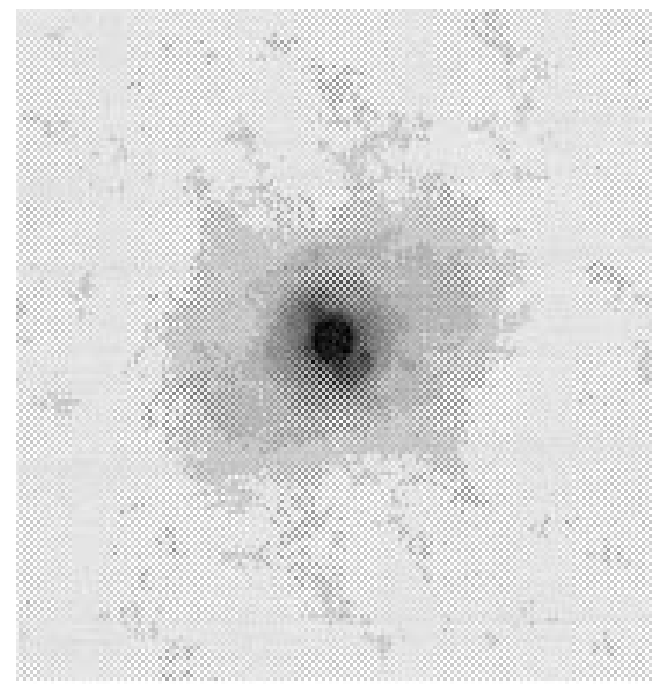

Fig. 11. The HRG 54103 processed image after high-pass filtering as transfer function

We have adapted this relationship by adopting the center of the principal object (the most prominent galaxy, but the center of the frame may also be carefully used) as the origin of coordinates of the image plane, and no reference wave has been considered. The hologram so constructed is the new transfer function.

Figure 14 shows the results obtained with the highpass filtered hologram as a transfer function. This image has additionally been processed with a gaussian filter to enhance the structure of the edge-on dust disk of NGC 5193A. The disk extends to the top and then to the left of the image, spanning a broad region above both galaxies. The "empty" region around the nuclei are artifacts introduced by the gaussian filter chosen. The reader is referred to Fig. 5 for comparison.

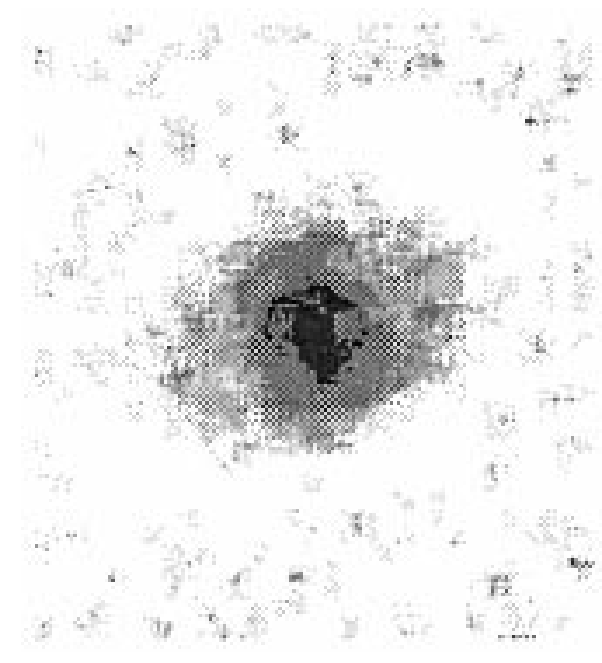

Fig. 12. High-pass filtered image of HRG 54103 - see text

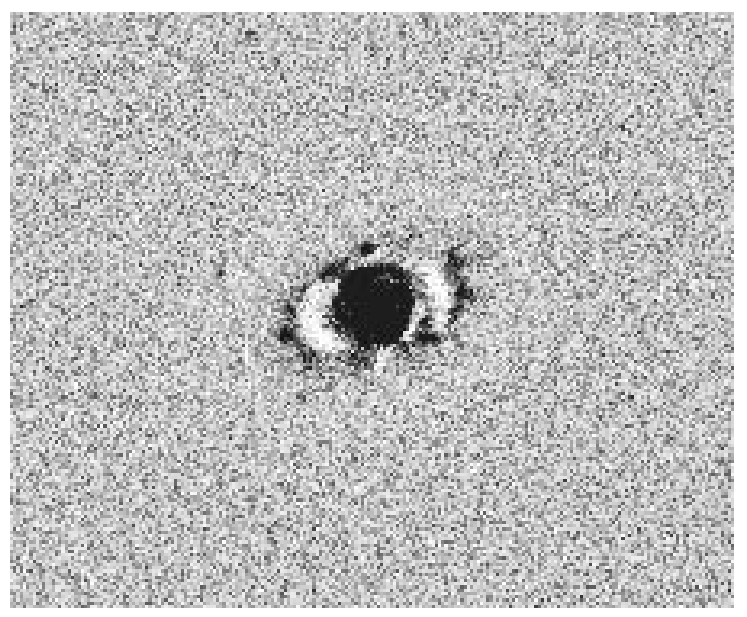

Fig. 13. The residual of the subtraction of a $15 \times 15$-pixel median image from the original image of HRG 54103

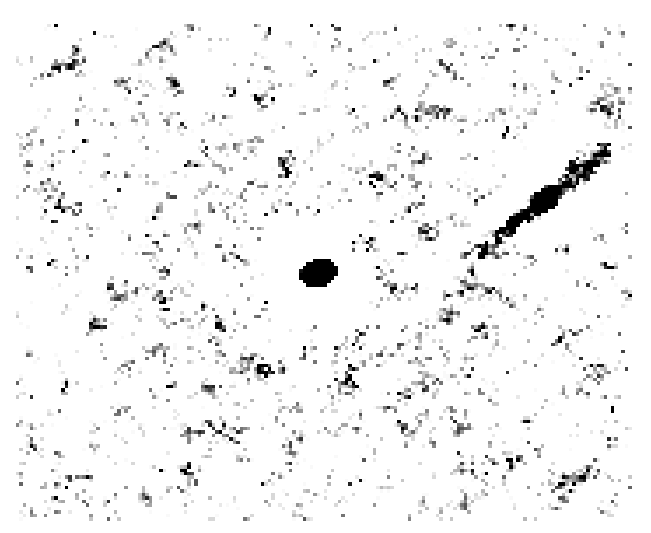

Fig. 14. The image of NGC 5193/5193A after the application of a Fourier hologram and gaussian filter. The edge-on dust disk and the arm have been enhanced 
Another experiment was to create a low-pass filtered hologram, to apply it to the image of NGC 5761, and then subtract the result from the original (see Fig. 15). The spikes discussed previously (Figs. 1, 2, and 3) have been strikingly highlighted. Again, the outer arm of ESO 580-G38 can be seen.

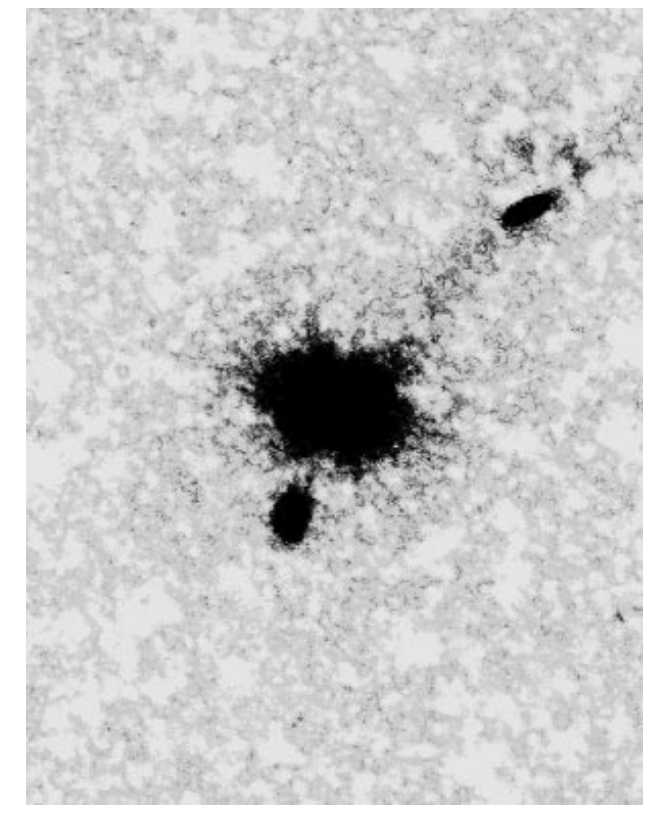

Fig. 15. Residual image of the subtraction of the low-pass filtered Fourier hologram of NGC 5761 from the original image. The radial spikes have been highly enhanced

In the case of HRG 54103, we have also made the difference between the original image and that obtained by the use of a low-pass hologram as transfer function (Fig. 16). Two satellites projected onto the bulge are discernible, both with different sizes than in Fig. 11. No diagnostics about the nucleus can be done due to the resolution of the image. With this contrast, the upper plume is not apparent.

\subsection{Background-suppressed filtering}

In an early and beautiful article, Sulentic \& Lorre (1984) demonstrated the potential of image processing. They stated that "new techniques are being created all the time by astronomers with special problems to solve". This is only partially true: a lot of research is being conducted in the fields of artificial vision, signal and optical image processing, microscopy, and pattern recognition. Astronomers frequently make use of these ideas.

We have based the next experiment on the idea of background-suppressed filtering (Scholl 1995), where the object scene is enhanced by suppressing selected background features. The following mathematical relation

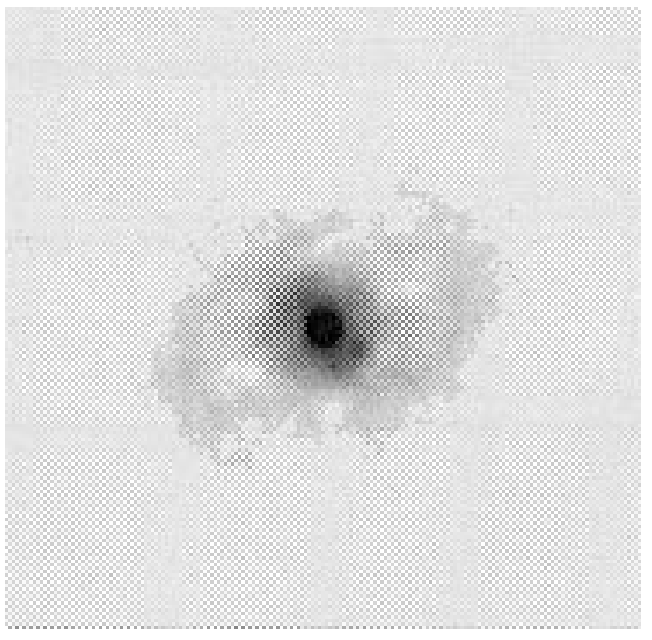

Fig. 16. After the application of the low-pass hologram as transfer function, the two satellites in the bulge of HRG 54103 are emphasized

$$
\begin{array}{r}
g_{f}^{\prime}(x, y)=F^{-1}\left\{\left[a_{\mathrm{s}}\left(f_{x}, f_{y}\right)-a_{\mathrm{b}}\left(f_{x}, f_{y}\right)\right]\right. \\
\left.\exp \left[-i \phi_{\mathrm{s}}\left(f_{x}, f_{y}\right)\right]\right\}
\end{array}
$$

is the background-suppressed scene, where $a_{\mathrm{s}}\left(f_{x}, f_{y}\right)$ and $a_{\mathrm{b}}\left(f_{x}, f_{y}\right)$ are the amplitudes of the Fourier transform of the scene and background, respectively; $\phi_{\mathrm{s}}\left(f_{x}, f_{y}\right)$ is the phase of the scene's Fourier transform.

In order to demonstrate the method's efficacy, an IRAF computer-generated image containing the letters $\mathrm{A}, \mathrm{O}$, and $\mathrm{L}$ as scene image (as in Fig. 5a of the work of Chen \& Chen 1995) and an equally-sized frame with the letter A only - the background image - have been used. The experiment succeeded in eliminating the letter A from the scene, as displayed in Fig. 17.

The next step then was to test this technique for its ability of differentiating galactic structures through the suppressing of selected patterns, either background or not. We have worked on our galaxies with satisfactory results (Faúndez-Abans \& de Oliveira-Abans 1998).

The reader is referred to the works of Baba et al. (1994); Frieden \& Bajkove (1996); Paranjape et al. (1994); Carnicer et al. (1994); Shi \& Ward (1995); Brasher \& Woodson (1996); Tom et al. (1996); Pluzhnik (1996); Thurnhofer \& Mitra (1996) and Yung \& Lai (1996) for other ideas on image identification, restoration, correlations, simulations, and noise subtraction.

\section{Discussion}

Working in the transform domain opens a fan of possibilities like: testing of several kinds of filters, perturbation of the signal of the transformed image (real and imaginary parts) to search for the occurrence of specific features, and the application of different scene object models. 

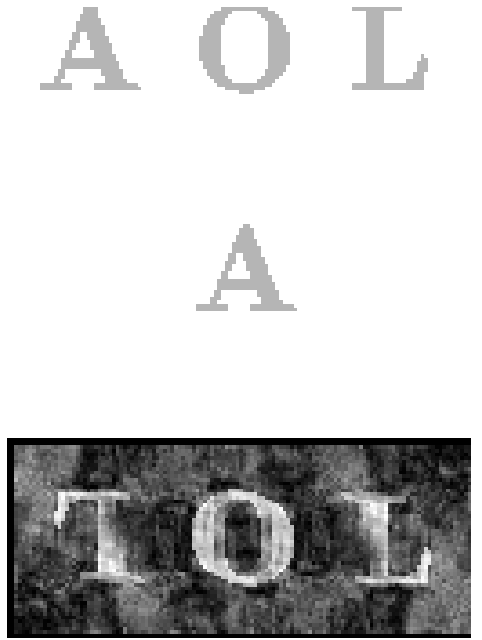

Fig. 17. Top: the scene image, middle: the background image to be suppressed, and bottom: the resultant frame

The reader is referred to the works of Mahalanobis et al. (1994); Javidi et al. (1994); Juvells et al. (1994); Javidi \& Wang (1995); Martucci (1996); Javidi \& Painchaud (1996). For analysis and restoration of direct images and spectra, there are the works of Brault \& White (1971); Lucy (1974); Högbom (1974) and Baade \& Lucy (1990). The case of Wiener filtering, for instance, does not take into account the spatially variant statistics (the stationary hypothesis) and is minimum mean-square-error constrained. The transfer function of the Wiener filter has a large magnitude at those spatial frequencies for which the signal power is large if compared to the noise power. These facts are partially responsible for the smooth look of the so restored images, in contrast to the human eye, which accepts more noise whenever associated to sharp intensity changes (STSDAS help page on "restore" and Arp \& Lorre 1976). Incomplete deconvolution due to the cutoff of certain high frequencies may form haloes around bright regions against darker background.

Deconvolution using the point spread function of frame stars in the field of the galaxies is also useful in the search for structures in galaxies (Zavatti et al. 1990). Some artifacts introduced during the deconvolution of images of E-S0 galaxies have been reported by Michard (1996), but this can happen during any step of the processing in the transform domain. Some other ideas about deconvolution techniques can be found in the works of Yaroslavsky \& Caulfield (1994) and Thiébaut \& Conan (1995).

The idea of computer-generated holograms is recent (Mori \& Ohba 1994; Nagashima 1996, and references quoted therein), and so is the digital recording and mathematical reconstruction of Fresnel holograms (Schnars \& Jüptner 1994). The computer-generated Fourier holograms of the present work are simple; they have been built from the original images to create a transfer function for feature enhancement. Problems of artifacts may be present, also because the traditional methods generally require many iterations of the Fourier and inverse Fourier transforms (see Gabel \& Liu 1970; Hauck \& Bryngdahl 1984; Ersoy et al. 1992 and Chang \& Ersoy 1993). See Schnars \& Jüptner (1994), Mugnier (1995), Dorsch et al. (1994), and Nagashima (1996) for interesting reading.

We have shown that the mixing of different procedures may be necessary to enhance morphological details of extended objects. Care must be taken when choosing the parameters so that artifacts are reduced to a minimum. Creating transfer functions and filtering images are easily and quickly performed in the STSDAS and IRAF environments. We believe that these operations performed in the transform domain may improve automated galaxy classification. Improved filtering techniques could also be useful to enhance cooling flows' signatures in galaxy clusters (see Friaça 1996 for detection problems), and, why not, contribute to the effort of the primeval galaxy search (see Pritchet 1996).

Detailed studies on HRG 54103 will be presented in a forthcoming paper (Faúndez-Abans \& de Oliveira-Abans 1998).

Acknowledgements. This work has been partially supported by the Conselho Nacional de Desenvolvimento Científico e Tecnológico (CNPq, Brazil). This research has made use of the NASA/IPAC Extragalactic Database (NED) which is operated by the Jet Propulsion Laboratory, Caltech, under contract with the National Aeronautics and Space Administration. We would like to thank Dr. J. Sulentic for his comments and interesting suggestions to the early version of the manuscript.

\section{References}

Aoki S., Noguchi M., Iye M., 1979, in Photometry, kinematics and dynamics of galaxies, Evans D.S. (ed.). Austin: University of Texas Press, p. 411

Arp H., Lorre J.J., 1976, ApJ 210, 58

Baade D., Lucy L.B., 1990, ESO Messenger 61, 24

Baba N., Tomita H., Miura N., 1994, Appl. Opt. 33, 4428

Bates R.H.T., McDonnell M.J., 1989, in Image Restoration and Reconstruction, the Oxford Engineering Science Series. Clarendon Press, Oxford

Bow S.-T., 1984, Pattern recognition, Dekker M. (ed.). INC, New York

Brasher J.D., Woodson M., 1996, Appl. Opt. 35, 314

Brault J.W., White O.R., 1971, A\&A 13, 169

Buta R., 1995, ApJS 96, 39

Carnicer A., Vallmitjana S., Juvells I., Moneo J.R. de F., 1994, Appl. Opt. 33, 3070

Chang M.P., Ersoy O.K., 1993, Appl. Opt. 32, 3122

Chen X., Chen Z., 1995, Appl. Opt. 34, 879

Considère S., 1980, Ph. D. Thesis, Université de Paris VII

Considère S., Athanassoula E., 1982, A\&A 111, 28

Considère S., Athanassoula E., 1988, A\&S 76, 365

Cora S.A., Muzzio J.C., Vergne M.M., 1996, MNRAS 289, 253

Danver C.-G., 1942, Ann. Obs. Lund. No. 10

Dorsch R.G., Lohmann A.W., Sinzinger S., 1994, Appl. Opt. 33,869 
Ersoy O.K., Zhuang J.Y., Brede J., 1992, Appl. Opt. 31, 6894

Faúndez-Abans M., Hertling G.P., Ramírez A.C., 1992, A\&AS 94, 245

Faúndez-Abans M., de Oliveira-Abans M., 1998 (in preparation)

Friaça A.C., 1996, IAGUSP VIII Advanced School of Astrophysics, Itatiaia, Brazil

Frieden B.R., Bajkova A.T., 1994, Appl. Opt. 33, 219

Gabel R.A., Liu B., 1970, Appl. Opt. 9, 1180

Harris W.E., Smith M.G., Myra E.S., 1983, ApJ 272, 456

Hauck R., Bryngdahl O., 1984, J. Opt. Soc. Am. A 1, 5

Högbom J.A., 1974, A\&AS 15, 417

Iye M., Okamura S., Hamabe M., Watanabe M., 1982, ApJ 256,103

Iye M. 1983, in Internal kinematics and dynamics of galaxies, Athanassoula E. (ed.), p. 153

Javidi B., Tang Q., Zhang G., Parchekani F., 1994, Appl. Opt. 33,6219

Javidi B., Wang J., 1995, J. Opt. Soc. Am. A 12, 2604

Javidi B., Painchaud D., 1996, Appl. Opt. 35, 318

Jedrzejewski R.J., 1987, MNRAS 226, 747

Juvells I., Carnicer A., Vallmitjana S., Campos J., 1994, J. Opt. 25, 35

Kalnajs A.J., 1974, in La dynamique des galaxies spirales, Colloque Intern. CNRS, No. 241, Weliachew L. (ed.), p. 103

Lorre J.J., Gillespie A.R., 1980, SPIE Conf. 264, Applications of Digital Image Processing to Astronomy, 123

Lucy L.B., 1974, AJ 79, 745

Mahalanobis A., Vijaya Kumar B.V.K., Song S., Sims S.R.F., Epperson J.F., 1994, Appl. Opt. 33, 3751

Marion A., 1991, in An Introduction to Image Processing. Chapman and Hall
Martucci S.A., 1996, Opt. Eng. 35, 119

Michard R., 1996, A\&AS 117, 583

Mori K., Ohba R., 1994, Appl. Opt. 33, 3111

Mugnier L.M., 1995, Appl. Opt. 34, 1363

Nagashima K., 1996, Opt. Eng. 35, 570

Paranjape R.B., Kabie T.F., Rangayyan R.M., 1994, Appl. Opt. 33, 2861

Pluzhnik E.A., 1996, Astron. Rep. 40, 129

Pritchet C.J., 1996, IAGUSP VIII Advanced School of Astrophysics, Itatiaia, Brazil

Reduzzi L., Rampazzo R., Sulentic J.W., Prugniel P., 1994, The Messenger, No. 75, 28

Schnars U., Jüptner W.P.O., 1994, Appl. Opt. 33, 179

Schnars U., Jüptner W.P.O., 1994, Appl. Opt. 33, 4373

Secker J., 1995, PASP 107, 496

Scholl M.S., 1995, Opt. Eng. 34, 887

Shi X., Ward R.K., 1995, J. Opt. Soc. Am. A 12, 47

Solomon J.A., Pelli D.G., 1994, Nat 369, 395

Sulentic J.W., Lorre J., 1984, Sky \& Telescope 67, 407

Sulentic J.W., Arp H., Lorre J., 1985, AJ 90, 522

Thiébaut E., Conan J.-M., 1995, J. Opt. Soc. Am. A 12, 485

Thurnhofer S., Mitra S.K., 1996, Opt. Eng. 35, 1863

Tom B.C., Lay K.-T., Katsaggelos A.K., 1996, Opt. Eng. 35, 241

Toney E., 1983, Image enhancement through clustering specification, Masters Thesis, Pennsylvania State University, University Park

Yaroslavsky L.P., Caulfield H.J., 1994, Appl. Opt. 33, 2157

Yung N.H.C., Lai A.H.S., 1996, Opt. Eng. 35, 1871

Zavatti F., Bendinelli O., Parmeggiani G., Palumbo G.G.C., Bignami G.F., 1990, ESO Messenger 61, 60 